\title{
Standardizing registry data to the OMOP Common Data Model: experience from three pulmonary hypertension databases
}

Patricia Biedermann ${ }^{1}$, Rose Ong ${ }^{1}$, Alexander Davydov², Alexandra Orlova², Philip Solovyev², Hong Sun', Graham Wetherill $^{3}$, Monika Brand ${ }^{1}$ and Eva-Maria Didden ${ }^{* *}$

\begin{abstract}
Background: The Observational Medical Outcomes Partnership (OMOP) Common Data Model (CDM) can be used to transform observational health data to a common format. CDM transformation allows for analysis across disparate databases for the generation of new, real-word evidence, which is especially important in rare disease where data are limited. Pulmonary hypertension $(\mathrm{PH})$ is a progressive, life-threatening disease, with rare subgroups such as pulmonary arterial hypertension (PAH), for which generating real-world evidence is challenging. Our objective is to document the process and outcomes of transforming registry data in PH to the OMOP CDM, and highlight challenges and our potential solutions.
\end{abstract}

Methods: Three observational studies were transformed from the Clinical Data Interchange Standards Consortium study data tabulation model (SDTM) to OMOP CDM format. OPUS was a prospective, multi-centre registry (20142020) and OrPHeUS was a retrospective, multi-centre chart review (2013-2017); both enrolled patients newly treated with macitentan in the US. EXPOSURE is a prospective, multi-centre cohort study (2017-ongoing) of patients newly treated with selexipag or any PAH-specific therapy in Europe and Canada. OMOP CDM version 5.3.1 with recent OMOP CDM vocabulary was used. Imputation rules were defined and applied for missing dates to avoid exclusion of data. Custom target concepts were introduced when existing concepts did not provide sufficient granularity.

Results: Of the 6622 patients in the three registry studies, records were mapped for 6457. Custom target concepts were introduced for PAH subgroups (by combining SNOMED concepts or creating custom concepts) and World Health Organization functional class. Per the OMOP CDM convention, records about the absence of an event, or the lack of information, were not mapped. Excluding these non-event records, 4\% (OPUS), 2\% (OrPHeUS) and 1\% (EXPOSURE) of records were not mapped.

Conclusions: SDTM data from three registries were transformed to the OMOP CDM with limited exclusion of data and deviation from the SDTM database content. Future researchers can apply our strategy and methods in different disease areas, with tailoring as necessary. Mapping registry data to the OMOP CDM facilitates more efficient collaborations between researchers and establishment of federated data networks, which is an unmet need in rare diseases.

Keywords: Pulmonary hypertension, Registry, Observational data, Common data model, Data mapping

${ }^{*}$ Correspondence: edidden@its.jnj.com

${ }^{1}$ Actelion Pharmaceuticals Ltd, Gewerbestrasse 16, CH-4123 Allschwil, Switzerland

Full list of author information is available at the end of the article

\section{Background}

Evidence generated from observational, real-world data can be highly insightful and is increasing in importance, particularly in rare diseases where information is 
limited [1-3]. The analyses of observational data, such as administrative claims databases, electronic healthcare records, or registries, offer the potential for patient and disease characterization, drug surveillance, and comparison of the effectiveness or safety of interventions [4-7]. The gathering and analyses of real-world data to provide real-world evidence has been identified as a major priority in the twenty-first Century Cures Act of 2016 $[8,9]$ and in the concept paper for the proposed Cures Act 2.0 [10] in the US, with the aim of accelerating drug development and innovation. The analyses of data from multiple healthcare data sources can be an efficient and cost-effective approach for evidence generation [11]; however, it is difficult to combine data without losing information because each database has its own original purpose, objectives, structure, and terminology. A logical solution to address this problem would be to store data in a standardized format, such as a common data model (CDM). A CDM is an informational model that allows transformation of data contained in different databases to a common format, in which all coding and vocabulary are pre-specified and standardized [12], and can be applied to all data irrespective of product or therapy area. Transforming data sources into CDM is a convenient way to allow analyses across multiple sources.

Several data networks have been established with a view to improving the standardization of observational healthcare data and to establish shared open-source tools that facilitate collaborative advancement of disease understanding and research. Examples include the Sentinel initiative of the US Food and Drug Administration (FDA) and the European Union Adverse Drug Reactions (EU-ADR), which were both developed to monitor medical products [13-15], as well as the European Network of Centres for Pharmacoepidemiology and Pharmacovigilance (EnCePP), the National Patient-Centred Clinical Research Network (PCORnet) and the Health Care Systems Research Network (HCSRN; formerly HMO Research Network) [16-18]. Similarly, the Observational Medical Outcomes Partnership (OMOP), a public-private partnership between the FDA, academic institutions, database owners, and pharmaceutical companies, and the subsequent Observational Health Data Sciences Informatics (OHDSI) collaborative aimed to utilize existing observational healthcare data for safety surveillance [1922]. Most of the above-mentioned networks have developed their own CDM to meet their aims.

Studies comparing different CDMs showed that the OMOP CDM met most of the desired criteria (including content coverage, integrity, flexibility, ease of querying, standards compatibility, and ease/extent of implementations, privacy and linkage) for data sharing and across use cases [23, 24]. All other investigated CDMs (FDA
Mini-Sentinel/Sentinel, PCORnet, and the Clinical Data Interchange Standards Consortium study data tabulation model (SDTM) - which is the established standardized format and organization of clinical trial data [25]) scored lower for observational healthcare data than the OMOP $\mathrm{CDM}$ for content coverage, cost or clinical outcome measures, data linkage and/or case definition [23, 24]. Advantages of standardizing to the OMOP CDM include that users do not need to understand all database-specific schema details, firmly controlled terminology exists making datasets within the OMOP CDM comparable, and the concepts are freely available for researchers to access. Furthermore, in a replication analysis it has been reported that up to $80 \%$ less programming time was required in OMOP CDM than in the raw data [26].

Pulmonary hypertension $(\mathrm{PH})$ is a progressive and ultimately fatal disease, which is classified into five clinically diverse groups, including the rare subgroups pulmonary arterial hypertension (PAH) and chronic thromboembolic pulmonary hypertension (CTEPH) [27]. In $\mathrm{PH}$, there are a number of recently completed and currently ongoing registries, which are formatted to the SDTM. Registries are vital sources of information that can provide insight into the characteristics and longitudinal trends of a specific patient population $[28,29]$. The OPsumit ${ }^{\circledR}$ USers (OPUS) registry (NCT02126943) was a prospective drug registry initiated in response to an FDA post-marketing requirement to characterize the safety profile of the endothelin receptor antagonist, macitentan, in a real-world setting. The OPsumit ${ }^{\circledR}$ Historical USers (OrPHeUS) cohort study (NCT03197688) was a retrospective medical chart review designed to supplement OPUS with data from additional patients to achieve the necessary sample size for the primary outcome. EXPOSURE is an ongoing, international, multicentre, prospective, real-world, observational study (EUPAS19085) in patients with $\mathrm{PAH}$ following marketing authorization of the IP-receptor agonist, selexipag, by the European Medicines Agency (EMA). To date, predominantly large administrative claims or electronic medical records databases have been converted to a CDM $[6,7,26,30,31]$, while the mapping of registries has been seldom undertaken and presents additional challenges compared with coded databases. One study has mapped the German PH registry to the OMOP CDM from source data in Microsoft Access with limited details [32] and a second study has described the harmonization of data from the USbased SEARCH diabetes registry and the observational, multi-centre youth diabetes registry of patients in India to the OMOP CDM; however, the standardization process itself was not described [33].

The SDTM is the standard format used for clinical trial data and while it defines a standard structure for 
its tables and its framework allows flexibility, this structure is achieved through the wider availability of tables and columns following suggested patterns and these are tailored to clinical trial data. In contrast, the core structure of the OMOP CDM is fixed and the number of tables, and their respective fields, are finite, which allows for easier and consistent application development due to the underlying rigidity of the data model. Moreover, the OMOP CDM was designed for observational data (including claims and electronic healthcare records), and a number of observational healthcare databases have already been mapped to OMOP CDM, including a $\mathrm{PH}$ registry [30-32, 34-36]. In addition, a study comparing the utility of various CDMs for the purposes of comparative effectiveness research found the OMOP CDM to be the best-suited [37]. Importantly, in recent years, there has been evolution towards federated networks that foster research using disparate databases and are therefore in need of standardised data assets. Federated data networks, such as the European Health Data Evidence Network (EHDEN) [38], are considered as the future for research collaboration and real-world evidence generation in rare diseases. Therefore, the mapping of $\mathrm{PH}$ registry databases to the OMOP CDM allows researchers to use a wider base to generate more robust conclusions, as they can perform analyses across the many disparate real-world data assets available in OMOP CDM format. This can represent a particular advantage in a disease like $\mathrm{PH}$, as it is comprised of rare and diverse subgroups $[1,27]$. The data available on $\mathrm{PH}$ subgroups are limited; databases of patients with $\mathrm{PH}$ and its subgroups are often small and from disparate regions, without the possibility of readily pooling the data. We aim to describe our experience in transforming data from the OPUS, OrPHeUS and EXPOSURE observational studies from the SDTM to the OMOP CDM, and to highlight the benefits and challenges to this novel process.

\section{Methods}

\section{Data sources}

Three registries, formatted to the SDTM, were converted to the OMOP CDM, and are summarized in Table 1. For all datasets, the raw data are available in SDTM format before patients are excluded based on eligibility criteria. The SDTM format is determined by the design of the study and case report form (CRF), for example, tick boxes or free text and mandatory or optional fields. Therefore, all events are captured in SDTM, including those performed (e.g., 'yes' selected, or box ticked) and not performed (e.g., 'no' selected, or box left unticked), since it is of interest to these studies whether an assessment was performed. The flexibility of free text and optional fields means that there are sometimes missing or partially missing data in the SDTM.

The OPUS, OrPHeUS and EXPOSURE registries were reviewed and approved by the relevant ethics committee/

Table 1 Overview of the OPUS, OrPHeUS and EXPOSURE databases

\begin{tabular}{|c|c|c|c|}
\hline & OPUS & OrPHeUS & EXPOSURE \\
\hline Study identifier & NCT02126943 & NCT03197688 & EUPAS19085 \\
\hline Study type & $\begin{array}{l}\text { Multi-centre, prospective drug registry } \\
\text { of PH patients newly treated with } \\
\text { macitentan }\end{array}$ & $\begin{array}{l}\text { Multi-centre, retrospective medical } \\
\text { chart review of PH patients newly } \\
\text { treated with macitentan }\end{array}$ & $\begin{array}{l}\text { Multi-centre, prospective cohort study } \\
\text { of patients with PAH newly treated with } \\
\text { either selexipag or any other PAH- } \\
\text { specific therapy }\end{array}$ \\
\hline Region & USA & USA & Europe and Canada \\
\hline Date ranges & Apr 2014 - Apr 2020 & Oct 2013 to Mar 2017 & Sept 2017 - ongoing ${ }^{\text {a }}$ \\
\hline Patients in database, $\mathrm{N}$ & 2722 & 3142 & 758 \\
\hline $\begin{array}{l}\text { Age at enrolment in } \\
\text { database, mean (SD), } \\
\text { years [N] }\end{array}$ & $\begin{array}{l}60.93(14.15) \\
{[N=2682]}\end{array}$ & $\begin{array}{l}60.21(15.06) \\
{[N=3060]}\end{array}$ & $\begin{array}{l}59.96(15.07) \\
{[N=752]}\end{array}$ \\
\hline \multicolumn{4}{|l|}{ Gender, \% } \\
\hline Female & 71.6 & 72.2 & 68.6 \\
\hline Male & 26.9 & 25.2 & 31.3 \\
\hline Missing & 1.4 & 2.5 & 0.1 \\
\hline \multicolumn{4}{|l|}{ Source data format } \\
\hline Conditions & MedDRA and free text & MedDRA and free text & MedDRA and free text \\
\hline Drugs & WHODrug and free text & WHODrug and free text & WHODrug and free text \\
\hline
\end{tabular}

${ }^{a}$ Data cut-off for this analysis was 30 Nov 2019

Percentages might not add up to $100.0 \%$ due to rounding

MedDRA Medical Dictionary for Regulatory Activities; OPUS OPsumit ${ }^{\circledR}$ USers OrPHeUS OPsumit ${ }^{\circledR}$ Historical USers; $P H$ pulmonary hypertension; $P A H$ pulmonary arterial hypertension; SD standard deviation; USA United States of America; WHODrug World Health Organization Drug Dictionary 
Institutional Review Boards for each respective database. Patients in OPUS and EXPOSURE provided informed consent for their protected, anonymized health information to be stored in a computer database and analysed by researchers and healthcare professionals, and for the results of these analyses to be published. The requirement for informed consent was waived for OrPHeUS to enable inclusion of patients who had died in this retrospective observational study.

\section{OMOP CDM}

The OMOP CDM is a patient-centric model, meaning that every clinical event has, at a minimum, a patient identifier (ID number guaranteeing anonymity) and a date. This also allows healthcare events to be viewed over a given time horizon for each individual [22, 37]. Table 2 defines the key terms used in OMOP CDM. Patient and medical information are organized into 'domains' in the OMOP CDM, which are stored in domain-specific tables and fields, and examples include a drug domain or condition domain. In turn, these domain-specific tables are populated with 'standard concepts', which have a unique domain assignment dictating the table that it is recorded in $[22,37]$. In the OMOP CDM, the content of each patient record is transformed to the machinereadable format so that they are represented as 'concepts', which are stored in CONCEPT table $[22,37]$. The tables within the OMOP CDM contain equivalent information recorded in multiple ways at once: as a 'source value', a 'source concept,' and as a 'standard concept' (Table 2) [22, 37]. The OMOP standardized vocabulary is a common repository of all OHDSI-supported vocabularies and ensures standardization (Table 2) [22, 37]. The standard tables contained in the OMOP CDM are shown in Fig. 1.

\section{Sample selection}

In order to map the data in the registries to the OMOP CDM, patients with important data completely missing (such as birth year, or treatment start and end dates) were excluded as well as those who violated inclusion/exclusion criteria of the original registry (but were mistakenly enrolled before being excluded from further data entry, and thus had partial data in the SDTM database). These patients were not part of the target population for the registry. Duplicate records for the same patient within a registry were also excluded. For the OrPHeUS and OPUS finalized studies, mapping to OMOP CDM was performed initially in September 2019 and with the final data mapped in October 2020. The EXPOSURE database was first mapped in August 2019, with a refresher in December 2019. An annual data refresh is planned for EXPOSURE, which is an ongoing study.

\section{Mapping methods}

The tools and programs used for the mapping process are summarized in the Supplementary Appendix. The following roles were required for expertise in the different parts of the mapping process: project manager; epidemiology data analyst; biostatistician, developer; tester; medical terminologist; medical expert; source data expert, and observational data scientist.

An overview of the 7-phase process of mapping registry data in the SDTM format to the OMOP CDM is shown in Fig. 2 and further details are in the Supplementary Appendix. In the first, pre-analysis phase,

Table 2 Definitions of OMOP CDM terminology

\begin{tabular}{|c|c|}
\hline & Definition \\
\hline Standardized vocabulary & A common repository of all terminologies used within the OMOP CDM consolidated into a common format \\
\hline Concept & A term defined in a medical terminology \\
\hline Source values & $\begin{array}{l}\text { The verbatim representation of an event record in the source data using the original codes from public code systems (e.g. } \\
\text { ICD, National Drug Code, Current Procedural Terminology 4th edition) or locally controlled vocabularies that are not used in } \\
\text { analysis and only provided for convenience and quality purposes }\end{array}$ \\
\hline Source concepts & $\begin{array}{l}\text { Source concepts represent the terms in the common healthcare terminology systems that were used in the source data- } \\
\text { base, and are often found in the OMOP vocabularies as non-standard concepts }\end{array}$ \\
\hline Standard concepts & $\begin{array}{l}\text { Standard concepts are the respective target concepts that define the unique meaning of a clinical entity and are typically } \\
\text { drawn from existing public terminologies such as SNOMED or created within OMOP as extension concepts if no suitable } \\
\text { target is available }\end{array}$ \\
\hline Custom concepts & Clinical terms that are custom generated for information that is not represented in the standardized vocabulary \\
\hline Classification concepts & $\begin{array}{l}\text { These are non-standard concepts that do not represent the data in the OMOP CDM, but instead are part of the standard } \\
\text { concepts hierarchy, and can be used for hierarchical queries to search for a certain concept. They can be used for analysis, } \\
\text { but they have no full equivalent 'maps to' links to standard concepts. Hence, classification concepts, such as MedDRA terms, } \\
\text { require a degree of manual mapping }\end{array}$ \\
\hline
\end{tabular}

ICD International Classification of Disease; MedDRA Medical Dictionary for Regulatory Activities; OMOP CDM Observational Medical Outcomes Partnership common data model; SNOMED Systematized Nomenclature of Medicine Clinical Terms 


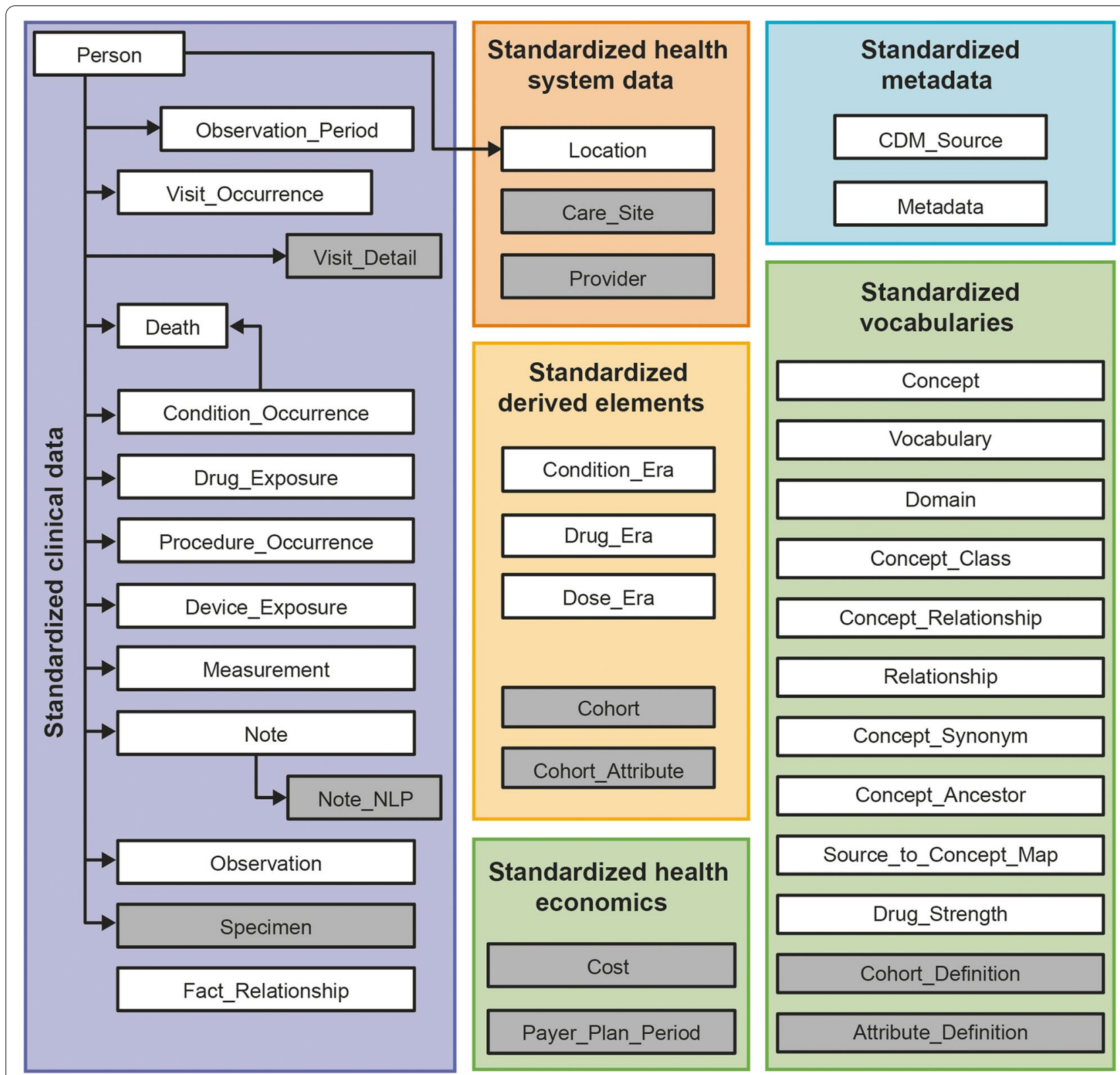

Fig. 1 Standardized tables and vocabularies available in the OMOP CDM. Tables and vocabularies in grey were not populated/used during this analysis. Table adapted from The Book of OHDSI, chapter 4 [39]. OMOP CDM, Observational Medical Outcomes Partnership Common Data Model

source documentation and the SDTM were reviewed and a list of questions to discuss with the source data experts was prepared. From this, initial matching of source tables to OMOP CDM tables was performed and a list of custom vocabularies and sets of values to be custom mapped by medical experts was determined. The custom mapping of source values was performed when source concepts and/or codes were not available with equivalent standard concepts in the standardized OMOP CDM vocabularies. A specific example of mapping source tables to OMOP CDM tables is shown in Fig. 3. Currently, all-level MedDRA terms, which are used in the OPUS, OrPHeUS and EXPOSURE databases, are not an OHDSI-supported vocabulary but are considered 'classification concepts' (Table 2) in OMOP. As classification concepts, MedDRA terms have no full equivalent 'maps to' links to standard concepts, meaning that the MedDRA codes have no direct translation to OMOP standardized vocabulary for conditions and laboratory data (SNOMED, LOINC) and, thus, a 


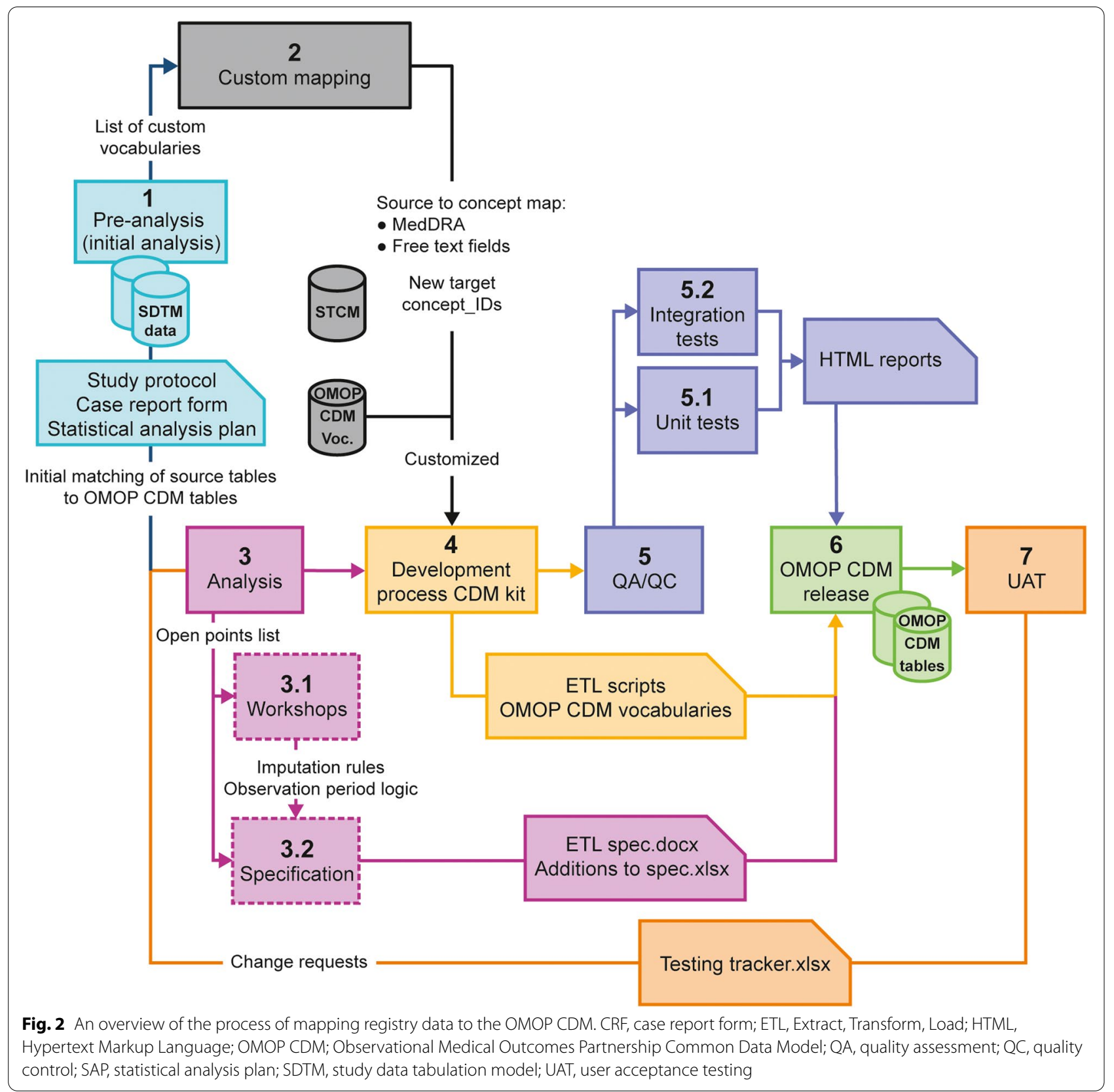

degree of manual mapping was required. This process is described in further detail in the results.

As the OMOP CDM requires complete dates, imputation rules were developed where this information was missing. Complete or partial (only year or year and month available in original record) missing dates for adverse events, laboratory tests, medical history, medications, hospitalizations, assessments and procedures were imputed. In addition, custom concepts were generated to capture information such as adverse event severity and causality, for which the OMOP CDM lacks a way to capture concisely.

Data refreshes (e.g., for EXPOSURE) involve similar steps to the original mapping. The version of the OMOP vocabulary is updated, if required, and the source data are closely examined for any significant changes compared with the initial data set. Unknown source codes are extracted, as well as any codes that have changed their description, and mapping is provided for these codes. The mapping is aligned to the current conventions and 


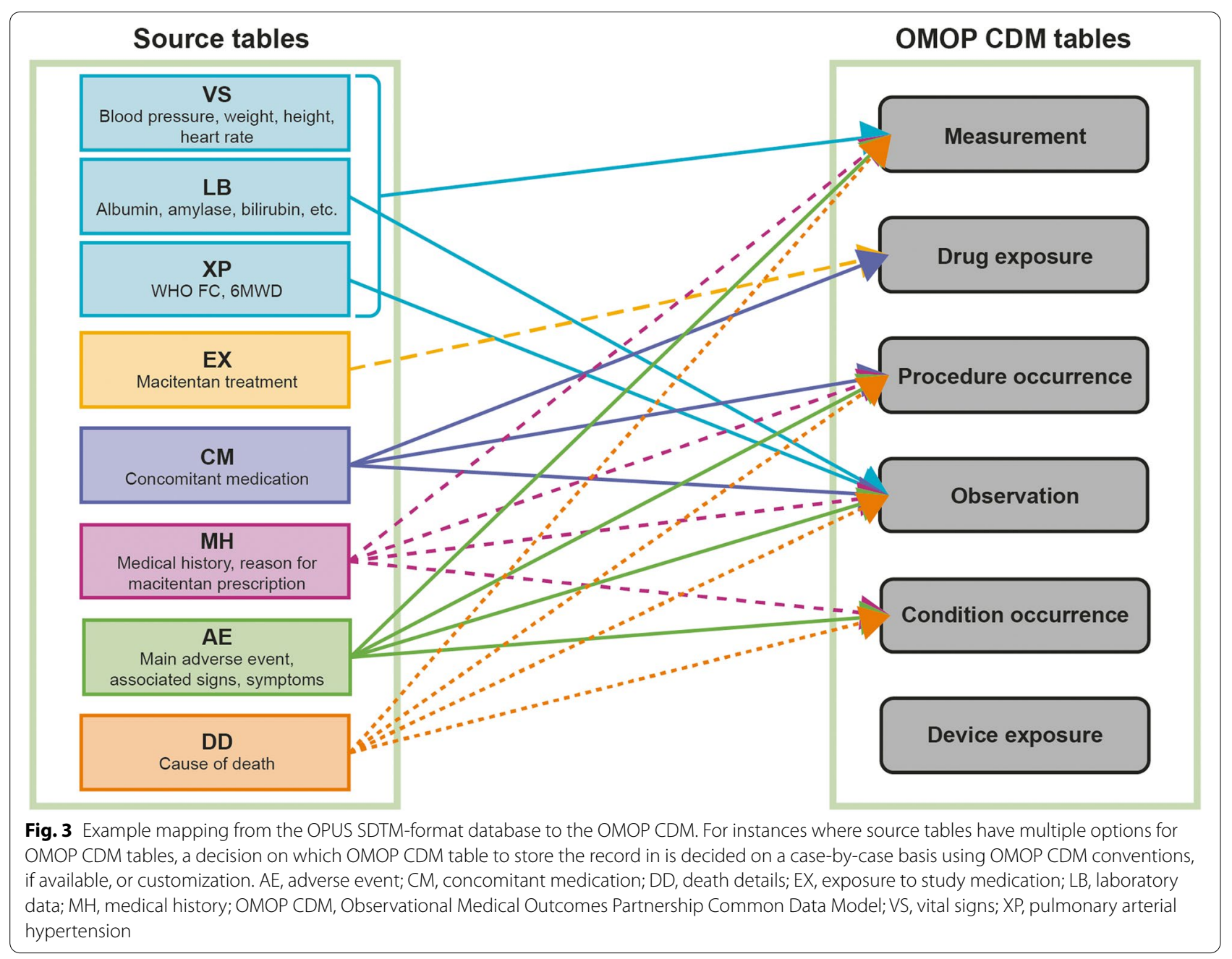

ETL specifications, and the custom concepts, CONCEPT_RELATIONSHIP and CONCEPT_ANCESTOR tables are rebuilt. Finally, the ETL code is adjusted and run on the new data.

\section{Quality assessment methods}

Descriptive summary statistics were obtained for the SDTM and OMOP CDM data sets, programmed in $\mathrm{R}$ (version 3.4.3), to compare consistency between source data and mapped data.

\section{Results}

\section{Database-specific information}

Of the 2722, 3142, and 758 patients in OPUS, OrPHeUS, and EXPOSURE, respectively, a total of 132 were excluded for violating original study inclusion/exclusion criteria and 33 were excluded due to OMOP CDM mapping conventions (Table 3). To note, the number of patients who were excluded due to violation of the
Table 3 Data exclusion in the mapping process to the OMOP CDM

\begin{tabular}{llll}
\hline & OPUS & OrPHeUS & EXPOSURE \\
\hline Patients in database, N & 2722 & 3142 & 758 \\
$\begin{array}{l}\text { Patients violating original } \\
\text { study inclusion criteria, } \mathrm{n}(\%)\end{array}$ & $46(1.7)$ & $85(2.7)$ & $1(0.1)$ \\
$\begin{array}{l}\text { Patients excluded during } \\
\text { mapping to OMOP CDM, } \mathrm{n}\end{array}$ & $2(0.1)$ & $25(0.8)$ & $6(0.8)$ \\
$\begin{array}{l}\text { (\%) } \\
\text { Total patients mapped, } \mathrm{n}(\%)\end{array}$ & $2674(98.2)$ & $3032(96.5)$ & $751(99.1)$ \\
\hline
\end{tabular}

OMOP CDM Observational Medical Outcomes Partnership common data model; OPUS OPsumit ${ }^{\circledR}$ USers; OrPHeUS, OPsumit ${ }^{\circledR}$ Historical USers

original study inclusion/exclusion criteria was lower for EXPOSURE as all but one patient who were mistakenly enrolled in EXPOSURE were deleted from the electronic data capture prior to transfer of data to the SDTM database. The total number of mapped patients was 6457 
(Table 3). The main challenges and solutions, described herein, are summarized in Table 4.

\section{Applied imputation rules}

Workshops were scheduled with the registry study team members to define imputations rules, with our highest priority being to keep imputation rules as consistent as possible across the three registries. Existing imputation rules were used if available and appropriate (e.g., from the statistical analysis plan of the individual registry), and new imputation rules were developed by considering the unique study design and the CRF structure. Imputation rules were mainly developed based on the following four strategies: i) extraction of dates from free text fields (in instances where the date and timestamp are stored in the database as a text value); ii) usage of time points available in the SDTM (e.g., 'before patient discontinuation' or 'within 3 months of baseline'); iii) imputation based on the previous interval of taking a certain drug; iv) comparison of the year and/or month of a medical event with pre-defined reference time points such as date of death, drug initiation or end date, date of last available information or last follow-up visit, and end of study date.

For example, laboratory test dates were imputed as follows: if the SDTM time point for drug initiation was present and had, for example, the same month and year as the laboratory test date then the missing date was imputed with the drug initiation date. If the laboratory test time point was the last available information before study end then the missing date was imputed with either the drug end date or study end date, whichever occurred first. In addition, imputation of drug initiation dates was performed as follows: if the day and/or month was missing and the year was available, then the missing date was imputed with the first day of that month (for missing day only) or 1 January (for missing day and month) for the same year. However, if this date was before the end date of the previous drug interval, then the drug initiation date was imputed with the end date of the previous drug plus 1 day.

Furthermore, for patients with recorded death in the database, but with a partly or completely missing date of death, the date of death for patients was imputed based on several algorithms. Firstly, the date of death may have been available in the following sources: the study CRF, the drug safety database or as the date of an adverse event with a fatal outcome; if available, a partially missing date (day, month and/or year) was gathered from these sources. Secondly, if several possible dates from the same patient had the same number of missing parts (day and/or month), then the following hierarchy was applied: death details from the study CRF; adverse events with a fatal outcome from the study CRF; death details from the drug safety database. Finally, in cases where the date of death had still not been determined, it was imputed with the date of last available information. For partially missing dates where the imputed death date occurred prior to the date of last available information, then the imputed death date was replaced with the date of last available information.

\section{Mapping process and customization}

Some of the registry data captured in SDTM format could not be fully accommodated in the OMOP CDM design, and had to be stored in the OMOP CDM without adjustment of additional tables or fields. Table 5 contains the most frequent cases and ways in which this information was transferred and stored in the OMOP CDM.

To map MedDRA codes to standard concepts, mapping automation from the unified medical language system to

Table 4 Summary of the main challenges involved in mapping registry data to the OMOP CDM, and their solutions

\begin{tabular}{|c|c|}
\hline Challenge & Solution \\
\hline Mapping incomplete dates & Create standardized imputation rules to 'restore' missing dates \\
\hline Mapping of MedDRA codes (classification concepts) to standard concepts & $\begin{array}{l}\text { Automated mapping from the unified medical language system to the ICD- } \\
\text { 10, ICD-10-Clinical Modification or SNOMED vocabulary with crosslinks and } \\
\text { name matching, followed by further expert review and additional manual } \\
\text { mapping }\end{array}$ \\
\hline Mapping of free text e.g. from medication tables & $\begin{array}{l}\text { Free text extracted, custom mapped and contextualized via CONCEPT_ } \\
\text { RELATIONSHIP and CONCEPT_ANCESTOR tables }\end{array}$ \\
\hline Mapping of non-existing OMOP vocabulary e.g. PH subgroups & $\begin{array}{l}\text { Used either a combination of SNOMED concepts (e.g. PAH and underlying } \\
\text { cause), or a new custom concept (e.g. drug- and toxin-induced PAH) }\end{array}$ \\
\hline $\begin{array}{l}\text { Mapping information that is related to another piece of information e.g. } \\
\text { severity of an event }\end{array}$ & $\begin{array}{l}\text { Separate clinical facts are stored in their appropriate domain and a link is } \\
\text { added in the FACT_RELATIONSHIP table }\end{array}$ \\
\hline Capturing the information that a procedure was not performed & $\begin{array}{l}\text { This information was excluding from mapping, per the OMOP convention, } \\
\text { but is planned for mapping in a future update }\end{array}$ \\
\hline
\end{tabular}

ICD International Classification of Disease; MedDRA Medical Dictionary for Regulatory Activities; OMOP CDM Observational Medical Outcomes Partnership common data model; SNOMED Systematized Nomenclature of Medicine Clinical Terms 


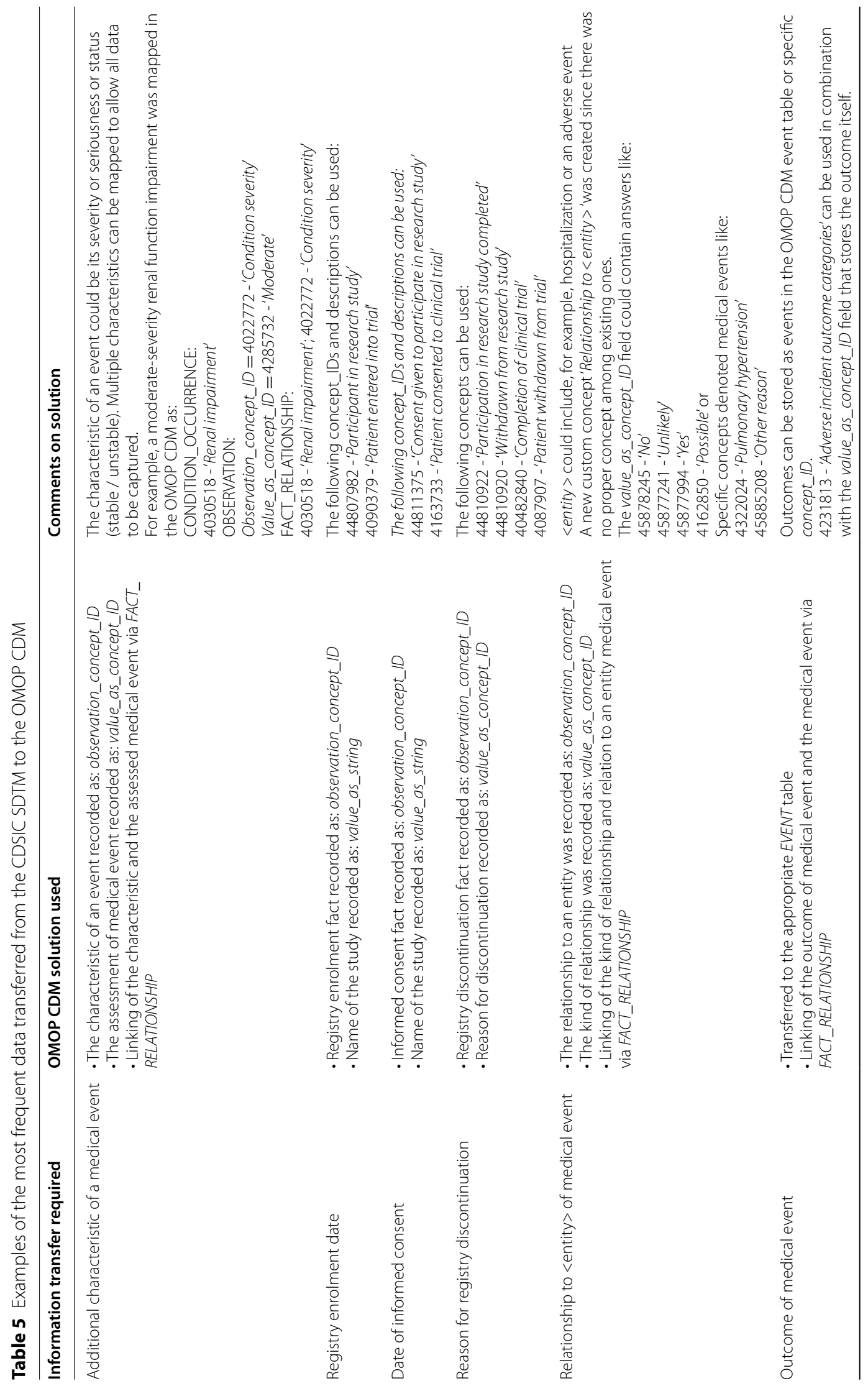




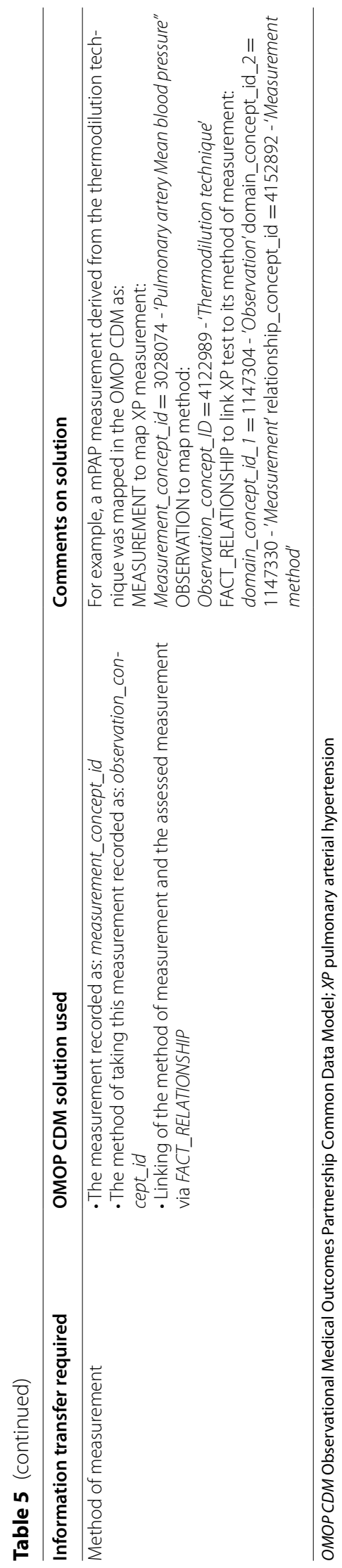


the ICD-10/ICD-10-Clinical Modification or SNOMED vocabulary with crosslinks and name matching, with further expert review and additional manual mapping were performed. Concomitant and study medication data were encoded in the source data using the World Health Organization Drug Dictionary (WHODrug) vocabulary, which is not an OHDSI-supported vocabulary. In addition, the free text from medication tables as well as, for example, adverse events, laboratory tests, reasons for death, medical history, clinical events (that were not MedDRA coded) were required to be extracted and custom mapped, and contextualized via CONCEPT RELATIONSHIP and CONCEPT_ANCESTOR tables if needed.

OMOP standardized vocabulary was used during the mapping process, and custom concepts were only generated when granular information could not be accurately mapped with existing vocabulary (such as for the different subgroups of PAH). In these cases, either a combination of SNOMED concepts was used for differentiation, e.g., PAH associated with connective tissue disease required $\mathrm{PAH}$ plus connective tissue disease overlap syndrome, or for $\mathrm{PAH}$ associated with congenital heart disease, PAH plus history of surgically corrected congenital heart defect was required. When mapping was not possible, custom concepts were introduced, such as for the disease subgroups of drug- and toxin-induced PAH and $\mathrm{PH}$ with unclear and/or multifactorial mechanisms (Group 5). Similarly, the standardized vocabulary did not contain an appropriate target concept for WHO functional class, which is used to assess the severity of $\mathrm{PH}$, and custom concepts were introduced to address this.

All custom concepts were incorporated into the concept's hierarchy, with custom CONCEPT_RELATIONSHIP and CONCEPT_ANCESTOR tables. This process allows users to easily identify relationships between variables, e.g., between conditions and subclasses of conditions, which facilitates the identification of specific patient cohorts for further analysis. The custom concept 'drug- and toxin-induced $\mathrm{PAH}$ ', for instance, was integrated into the vocabulary as a descendent of the standardized standard concept 'PAH.' However, custom concepts operate only in the CDM instance, or group of instances, they were introduced to and cannot be used in the OMOP network studies. Once the OHDSI community identify the need for wide usage, these concepts can be integrated to the official OMOP vocabulary.

FACT_RELATIONSHIP tables were used to capture clinical information such as aetiology, and create links between treatment and an additional characteristic of an event, such as severity, reasons for dose change, hospitalization (causality), and the outcome of the adverse event. First, the separate clinical facts were stored in their appropriate domains, and second, the link between them was added in the FACT_RELATIONSHIP table.

With respect to the measurement and condition tables, the OMOP CDM does not differentiate between procedures or conditions that were not performed or not reported. The OMOP CDM convention is to only include events that have actually occurred; records about the absence of an event, or the lack of information, were, therefore, not mapped to the OMOP CDM.

\section{Quality assessments}

The mapping process resulted in limited data exclusion, but a considerable consolidation of information (Table 3); most information was able to be supported by the OMOP CDM, either directly, or indirectly, via customization.

For condition codes, 10,659, 4013, and 449 unique source values (MedDRA codes or unique wording) were in the OPUS, OrPHeUS and EXPOSURE databases, respectively (Table 6). In the mapping process, if source values were the same, but appeared in different wording, they were mapped to the same concept_ID; accordingly, only 3698, 2704 and 337 unique concept_IDs were included in the OMOP CDM as a result of crosslinking, name matching, and custom mapping, for the OPUS, OrPHeUS and EXPOSURE records, respectively (Table 6). Therefore, 65\% (OPUS), 33\% (OrPHeUS) and $25 \%$ (EXPOSURE) of source values for condition codes were redundant and mapped to an existing concept_ID during this consolidation process. In total, 199,165 unique source records for condition codes were mapped to 108,657 unique OMOP CDM records (Table 6). Similarly, for drug codes, 51,612 unique source records were mapped to 46,360 unique OMOP CDM records (Table 6).

The total percentage of excluded records from OPUS, OrPHeUS and EXPOSURE when mapping to the OMOP CDM was $35 \%, 7 \%$ and $52 \%$, respectively (Supplementary Tables 1-3). The high percentage of records excluded from the OPUS and EXPOSURE databases was due to the large number of records in the clinical events table that were either marked as 'unknown' or 'not occurred' (as a result of simply being left un-ticked and translated into SDTM as a record showing that the event did not occur) and, thus, were not incorporated, as per OMOP CDM convention. The proportion of records that were not mapped as a result of being 'unknown' or 'not done' or having 'not occurred' is shown in Supplementary Tables 4-6. When these non-occurring events and event records with information that was irrelevant to analyses were excluded from calculations, 4\% (OPUS), 2\% (OrPHeUS) and 1\% (EXPOSURE) of records were not mapped (Supplementary Tables 1-3). 
Table 6 Quality assessment results

\begin{tabular}{|c|c|c|c|}
\hline Condition codes & OPUS & OrPHeUS & EXPOSURE \\
\hline Unique source values in SDTM database, N & 10,659 & 4013 & 449 \\
\hline Unique concept_IDs in OMOP CDM, n & 3698 & 2704 & 337 \\
\hline Unique custom concept_IDs in OMOP CDM, n & 6 & 6 & 5 \\
\hline Database-specific unique custom concept_IDs ${ }^{\mathrm{a}}, \mathrm{n}$ & 0 & 0 & 3 \\
\hline Unique source records in SDTM database, N & 128,858 & 41,960 & 28,347 \\
\hline Unique OMOP CDM records, $\mathrm{n}$ & 62,048 & 41,993 & 4616 \\
\hline Unique source records, $\mathrm{n}$ & 55,789 & 41,608 & 4394 \\
\hline Drug codes & OPUS & OrPHeUS & EXPOSURE \\
\hline Unique source values in SDTM database, $\mathrm{N}$ & 2768 & 894 & 2496 \\
\hline Unique concept_IDs in OMOP CDM, n & 1514 & 640 & 1050 \\
\hline Unique custom concept_IDs in OMOP CDM, n & 4 & 4 & 0 \\
\hline Database-specific unique custom concept_IDs ${ }^{\mathrm{a}}, \mathrm{n}$ & 3 & 0 & 0 \\
\hline Unique source records in SDTM database, N & 26,276 & 16,498 & 8838 \\
\hline Unique OMOP CDM records, $\mathrm{n}$ & 23,368 & 15,169 & 7823 \\
\hline Unique source records, $\mathrm{n}$ & 23,083 & 16,005 & 7537 \\
\hline
\end{tabular}

${ }^{a}$ Unique custom concept_IDs that were only used on one database; OPUS and OrPHeUS used the same custom condition concept_IDs

The high percentage of records lost from the EXPOSURE database was due to the large amount of events in the clinical events table that were either marked as 'unknown' or 'not occurred' and were thus not incorporated, as per OMOP CDM convention

For OrPHeUS condition codes, the number of unique OMOP CDM records is higher than the number of unique source records in the SDTM database because (i) there are very few events that are marked as 'unknown' or 'not occurred', hence the vast majority of events are mapped to OMOP CDM and (ii) some of the source records can be mapped to more than one target concept_IDs. For example, source code MedDRA 10057688 - 'Catheter site discharge' is mapped to 4249456 - 'Complication of catheter' and 4183956 - 'Skin discharge'

For example: source code MedDRA 10057688 - Catheter site discharge is mapped to 4249456 - Complication of catheter and 4183956 - Skin discharge

OMOP CDM Observational Medical Outcomes Partnership Common Data Model; OPUS OPsumit ${ }^{\circledR}$ USers OrPHeUS, OPsumit ${ }^{\circledR}$ Historical Users

\section{Discussion}

In this study, novel approaches for converting SDTM data from three registry databases to the OMOP CDM were successfully developed and applied with limited deviation from the SDTM database content and very few records being excluded from mapping. This approach addresses a need for combining real-world data of patients with rare diseases for the purpose of evidence generation and could serve as reference for future researchers wishing to undertake similar data mapping projects.

Records from a total of 6457 patients in the OPUS $(n=2674)$, OrPHeUS $(n=3032)$, and EXPOSURE ( $n=751)$ databases were successfully mapped. In total, 34.7\% (OPUS), 67.4\% (OrPHeUS) and 75.1\% (EXPOSURE) of concept IDs for condition codes and 42.1\% (EXPOSURE), 54.7\% (OPUS) and 71.6\% (OrPHeUS) of those for drug codes could be and were mapped to the OMOP CDM. There are several characteristics of registry data that explain these percentages. For example, the flexibility of SDTM clinical trial tabulation (e.g., free text and optional fields) means there are often missing or partially missing dates (obliging imputation rules), and there are often also multiple entries with the same meaning, which either have to be consolidated to the same concept ID or have to be differentiated through the use of FACT_RELATIONSHIP tables. Therefore, not all condition and drug codes could be mapped as unique entries with similar or identical meanings in the SDTM (for example, 'unknown', 'unspecified,' 'not available' or 'na') mapped to the same concept_ID in the OMOP CDM. Consequently, there were fewer unique concept_IDs compared with unique source concept_IDs and this reflects that data are stored in a more consolidated form in the OMOP CDM, rather than data loss. Imputation rules and custom concepts were introduced to help achieve limited data exclusion. Imputation rules allowed for the retention of records with incomplete or missing dates, which are inherent to observational studies. Missing dates were especially prevalent in the OrPHeUS database wherein data were collected retrospectively from existing records in medical charts, which could be incomplete. In addition, data points occurring before study baseline often have partial or completely missing dates. All mapping and imputation rules were comprehensively documented, and were as consistent as possible across the registries. There will always be a certain level of imprecision associated with any imputation method but such imputations would be needed for analysis of the data regardless of whether data has been transformed to the CDM. Moreover, making imputation rules integral to 
the mapping process facilitates consistent analysis of the data.

Rare disease aetiologies are often not fully represented in medical vocabularies such as ICD and, as a result, custom concepts were required for this registry mapping process. In the OMOP CDM the standardized vocabulary is generally limited for rare diseases or rare subgroups of diseases, as the OMOP CDM was originally developed for electronic health records and claims data, which are based on ICD and other administrative codes [40, 41]. Standardized SNOMED concepts, like other administrative codes, are limited in their level of granularity and do not cover all rare diseases and disease subgroups. For instance, specific ICD and SNOMED terms do not exist for the different subgroups of PAH. To maintain $\mathrm{PH}$ disease classification and its clinically diverse subgroup information, custom concepts were introduced. An advantage of mapping multiple databases was that when a custom concept was introduced it could also be used for the mapping of subsequent databases. For example, the custom concept for drug- and toxin-induced $\mathrm{PAH}$ was first introduced in the OrPHeUS mapping, and was then also applied in the mapping of the OPUS and EXPOSURE databases. This process was already established by the HemOnc.org working group, which introduced vocabulary to standardize cancer conditions and treatments in the OMOP CDM that would allow structured analyses across registries [40, 41].

\section{Limitations}

Limitations of the OMOP CDM format and the mapping process should be considered in advance of future work in this area, and the present study aims to give researchers a better understanding of said challenges and limitations. One limitation that researchers should consider is that several study groups may be mapping similar databases within the same disease area to OMOP CDM at the same time and using their own imputation rules and custom concepts. If such groups decide to collaborate, workshop discussions may be required to reach an agreement and develop guidelines on the methods used. To minimise this, we propose that aetiologies, treatments and procedures for rare diseases should be incorporated into the OMOP standardized vocabulary. In addition, we propose that the OMOP standardized vocabulary should be updated to capture procedures that have conclusively not been performed.

Currently, the OMOP CDM mapping convention is to only translate events that have actually occurred (or procedures that have actually been performed) and, as a result, there were substantial differences in the number of source records that could be mapped to the CDM. For example, in the prospective OPUS and EXPOSURE databases, study sites were able to confirm if a condition was present with a tick (or absence of a tick if not) that is stored as 'yes' or 'no' in SDTM format. As a result of 'no' answers not being mapped to the OMOP CDM, only $43.3 \%$ and $15.5 \%$ of unique source records for conditions were mapped for OPUS and EXPOSURE, respectively. In contrast, $99.2 \%$ of conditions were directly mapped to OMOP CDM for OrPHeUS, in which data were collected retrospectively and generally only conditions that had occurred were captured in the database. While these examples indicate consolidated data storage in the OMOP CDM, it is well known that in clinical practice, assessments are often not systematically performed according to a strict protocol as in the case of randomized controlled trials [42] and it is sometimes advantageous to capture non-occurrence of events. Suggestions on how to retain this information (non-occurrence of events), when required, is available [43], but was not used in this work as recording the absence of events or conditions was not central to the aims of the original databases. It can provide valuable insights as, for example, the absence of an assessment could reflect real-world clinical practices or that the patient had severe disease, and this information will be mapped as part of our future work.

It is important to note that whilst we have performed quality checks on the mapped data, no research study has yet been performed on the mapped data in the OMOP CDM. Caution should be taken about the risk of oversimplifying interpretation of data and results after standardization. The mapping of OPUS, OrPHeUS, and EXPOSURE will allow us to perform many analyses in the common database structure. However, the choice of analysis should be carefully considered when dealing with multiple data sources in a CDM, as not all types of analysis can be feasibly or meaningfully applied to every data source or study design. It is crucial, therefore, to retain a certain level of nuance between databases that were originally designed for different purposes. For instance, disease-specific test results, such as the 6-min-walk distance (in metres) in PAH, should be analysed in diseasespecific registries rather than in general administrative databases where such results are often not available or are incomplete. Similarly, claims and other administrative databases cover broader patient populations and, thus, are best positioned to compare a $\mathrm{PH}$ population with patients who have other types of respiratory disease or with 'healthy' patients.

\section{Outlook and future work}

Our customized mapping methodology offers a foundation for future researchers wishing to map data from other registries or observational healthcare data sources. This foundation is of great value, particularly as no 
guidelines currently exist for the mapping of registry data to a CDM $[32,33]$. One group has described the mapping of a single German PH registry to OMOP CDM but the details of the mapping process are largely limited to the ETL process [32]. Their process is ongoing and has involved adding 34 new concepts, 54 concept relationships, and 68 concept ancestor entries to the vocabularies, thereby achieving $100 \%$ coverage of the $\mathrm{PH}$ Nice classification in OMOP [32]. Continued sharing of methodologies and experiences could help avoid duplication of effort, shape future guidelines and foster collaborations. As such, we plan to collaborate with other research groups and external stakeholders that are interested in mapping registry data.

Within the OHDSI network, a working group is currently developing guidelines that may create a consensus on how to transform clinical trial data [44] (e.g., define observation period, store severity/seriousness, trial outcomes, planned information, dispensed but not administered medication data). These guidelines are an expansion beyond the previous norm of the OMOP being claims-focused and are an important first step for the handling of data from research studies, and will be a great help, though may not capture all challenges in mapping registry data. Other useful resources for researchers are systematic analysis tools such as ATLAS, an open-source software, which allows users to perform cross-database observational analyses to generate real-world evidence from patient-level data, without writing any programming code [45], and can then be applied in each database mapped to the OMOP CDM format. To summarise, researchers wishing to map registries should consider these guidelines, along with the experience described herein and by other study groups.

Importantly, our results show that the OMOP CDM can be used to store registry data without loss of essential information, and therefore add to previous reports of OMOP CDM use for coded, structured data (such as claims of electronic health records data), to demonstrate the value of OMOP CDM. For ongoing database studies, we would recommend refreshing the mapping as new data become available, considering potential changes in the data itself as well as changes in how the data have been captured. These changes may require additional custom mapping (for example, if a new medication or procedure is introduced), new imputation rules and customization of existing ETL logic and this could take time to discuss and implement. Mapping refreshes may also be performed when updates of the OMOP vocabularies are released. A study analysing the mapped data from the OPUS, OrPHeUS and EXPOSURE databases is needed and could be compared with existing, real-world, $\mathrm{PH}$ datasets such as those from the PHederation Network, for example [46]. In addition, our future work will include a study comparing SDTM- and OMOP CDMbased analysis results to further understand the variances in the datasets and confirm if results from the mapped registries are robust and suitable for review by payers and researchers.

\section{Conclusions}

The success of our mapping process from the SDTM to the OMOP CDM in limiting exclusion of data has important implications for the generation of real-world evidence in both pulmonary hypertension and other rare diseases. Mapping registry data to the OMOP CDM facilitates more efficient collaborations between researchers and establishment of federated data networks, which is a major unmet need in rare diseases. Future researchers can apply our methods and solutions in mapping registry data in different disease areas with appropriate changes in customizations, as required by the nuances of the disease and/or study design.

\section{Abbreviations}

CDM: Common data model; CRF: Case report form; CTEPH: Chronic thromboembolic pulmonary hypertension; EMA: European medicines agency; EnCePP: European network of centres for pharmacoepidemiology and pharmacovigilance; ETL;: Extract, transform, load; EU-ADR: European union adverse drug reactions; FDA: Food and drug administration; HCSRN: Health care systems research network; ICD: International classification of diseases; LOINC: Logical observation identifiers names and codes; MedDRA: Medical dictionary for regulatory activities; OHDSI: Observational health data sciences informatics; OMOP: Observational medical outcomes partnership; OPUS: Opsumit ${ }^{\circledR}$ users; OrPHeUS: Opsumit ${ }^{\circledR}$ historical users; PAH: pulmonary arterial hypertension; PCORnet: National patient-centred clinical research network; PH: Pulmonary hypertension; SDTM: Standards consortium study data tabulation model; SQL: Structured query language; SNOMED: Systematized nomenclature of medicine..

\section{Supplementary Information}

The online version contains supplementary material available at https://doi. org/10.1186/s12874-021-01434-3.

Additional file 1.

\section{Acknowledgements}

Medical writing and editorial support were provided by Zoe Schafer, PhD and Richard McDonald of Watermeadow Medical, an Ashfield Company, part of UDG Healthcare plc, funded by Actelion Pharmaceuticals Ltd., a Janssen Pharmaceutical Company of Johnson \& Johnson. The authors would like to thank Erica Voss (Janssen Research \& Development, NJ, USA), Michael Kalfelz and Natalia Karataeva (Odysseus Data Services, Inc., MA, USA) for their input into this manuscript, as well as Vlad Korsik, M.D., Oleg Zhuk, M.D., and Alina Vaziuro, M.D. for their support with mapping vocabularies.

\section{Authors' contributions}

All authors contributed to the conception and design of the study. Across the concept review process: $M B$ and EMD were the strategic leads; $A D, A O$ and PS conducted the concept mapping and process development; and PB, MB and EMD reviewed the concept mapping. $\mathrm{RO}$ and $\mathrm{PB}$ supervised the mapping activities for each database as observational study scientists. $A D, A O, P S, E M D$ and PB performed the quality assessments of data. EMD, MB, HS, GW, PB, AD, 
$\mathrm{AO}$ and PS developed imputation rules and customized concepts. All authors contributed to the intellectual analysis and interpretation of the data, and critical revision of the manuscript. PB led the development of the manuscript. The author(s) read and approved the final manuscript.

\section{Funding}

This research was funded by Actelion Pharmaceuticals Ltd., a Janssen Pharmaceutical Company of Johnson \& Johnson, Allschwil, Switzerland.

\section{Availability of data and materials}

The datasets generated and/or analysed during the current study are not publicly available due to only the mapping process of existing datasets being described but further information are available from the corresponding author on reasonable request.

\section{Declarations}

\section{Ethics approval and consent to participate}

All methods were conducted in accordance with the relevant guidelines and regulations including the Declaration of Helsinki and approvals were obtained from the relevant ethics committees across all studies. The OPUS registry protocol (ID: AC-055-503) and informed consent forms were approved by the Quorum Review Institutional Review Board (IRB). The Western IRB (WIRB)-Copernicus Group approved the OrPHeUS chart review protocol (ID: AC-055-510) and waived the requirement for informed consent, to enable inclusion of patients who had died in this retrospective observational study. The EXPOSURE registry protocol (ID: AC-065A401) and informed consent forms were approved by the relevant local ethics committee of each site (Supplementary Table 7). Patients in OPUS and EXPOSURE provided informed consent for their protected, anonymised health information to be stored in a computer database and analysed by researchers and healthcare professionals, and for the results of this analysis to be published.

\section{Consent for publication}

Not applicable.

\section{Competing interests}

PB was contracted by Actelion Pharmaceuticals Ltd., a Janssen Pharmaceutical Company of Johnson \& Johnson. RO, HS, MB, and EMD are employees of Actelion Pharmaceuticals Ltd., a Janssen Pharmaceutical Company of Johnson \& Johnson. GW is an employee of Janssen-Cilag Ltd. AD, AO, and PS are employees of Odysseus Data Services, Inc., a contract research organisation that performed the OMOP CDM ETL work as part of a commercial engagement with Johnson \& Johnson.

\section{Author details}

'Actelion Pharmaceuticals Ltd, Gewerbestrasse 16, CH-4123 Allschwil, Switzerland. ${ }^{2}$ Odysseus Data Services, Inc., Cambridge, MA, USA. ${ }^{3}$ Janssen-Cilag Ltd, High Wycombe, UK.

Received: 21 December 2020 Accepted: 7 October 2021 Published online: 02 November 2021

\section{References}

1. Torbicki A, Bacchi M, Delcroix M, Farber HW, Ghofrani H-A, Hennessy B, et al. Integrating data from randomized controlled trials and observational studies to assess survival in rare diseases. Circ Cardiovasc Qual Outcomes. 2019;12(5):e005095.

2. Gagne JJ, Thompson L, O'Keefe K, Kesselheim AS. Innovative research methods for studying treatments for rare diseases: methodological review. BMJ. 2014;349:g6802.

3. Heikinheimo O, Bitzer J, García Rodríguez L. Real-world research and the role of observational data in the field of gynaecology - a practical review. Eur J Contracept Reprod Health Care. 2017;22(4):250-9.

4. Jensen PB, Jensen $L$ J, Brunak S. Mining electronic health records: towards better research applications and clinical care. Nat Rev Genet. 2012;13(6):395-405.
5. Hersh WR. Adding value to the electronic health record through secondary use of data for quality assurance, research, and surveillance. Am J Managed Care. 2007;13(6 Part 1):277-8.

6. Overhage JM, Ryan PB, Reich CG, Hartzema AG, Stang PE. Validation of a common data model for active safety surveillance research. J Am Med Inform Assoc. 2012;19(1):54-60.

7. Zhou X, Murugesan S, Bhullar H, Liu Q, Cai B, Wentworth C, et al. An evaluation of the THIN database in the OMOP common data model for active drug safety surveillance. Drug Saf. 2013;36(2):119-34.

8. Gabay M. 21st century cures Act. Hosp Pharm. 2017;52(4):264-5.

9. 21st Century Cures Act, 2016. Available at: https://www.govinfo.gov/ content/pkg/BILLS-114hr34enr/pdf/BILLS-114hr34enr.pdf [Last accessed November 2020]. In. United States of America.

10. 21st Centry Cures 2.0: Concept Paper, 2020. Available at: https://deget te.house.gov/sites/degette.house.gov/files/Cures\%202.0\%20Concept\% 20Paper_0.pdf [Last accessed November 2020]. In.

11. Cheng $\overline{H G}$, Phillips MR. Secondary analysis of existing data: opportunities and implementation. Shanghai Arch Psychiatry. 2014;26(6):371-5.

12. Hripcsak G, Duke JD, Shah NH, Reich CG, Huser V, Schuemie MJ, et al. Observational health data sciences and informatics (OHDSI): opportunities for observational researchers. Stud Health Technol Inform. 2015;216:574-8.

13. Food and Drug Administration: FDA's Sentinel Initiative: Transforming how we monitor the safety of FDA-regulated products. 2019.

14. Food and Drug Administration, Sentinel Initiative. Available at: https:// www.sentinelinitiative.org/ [Last accessed November 2020].

15. Oliveira JL, Lopes P, Nunes T, Campos D, Boyer S, Ahlberg E, et al. The EU-ADR web platform: delivering advanced pharmacovigilance tools. Pharmacoepidemiol Drug Saf. 2013:22(5):459-67.

16. The National Patient-Centered Clinical Research Network. PCORnet. Available at: https://pcornet.org/ [Last accessed November 2020].

17. Health Care Systems Research Network, HCSRN. Available at: http://www. hcsrn.org/en/ [Last accessed November 2020].

18. The European Network of Centres for Pharmacoepidemiology and Pharmacovigilance (ENCePP): Guide on Methodological Standards in Pharmacoepidemiology (Revision 8). 2020.

19. Observational Health Data Sciences and Informatics, OHDSI program. Available at: https://ohdsi.org/ [Last accessed November 2020].

20. Foundation for the National Institutes of Health, Observational Medical Outcomes Partnership (OMOP). Available at: https://fnih.org/what-wedo/major-completed-programs/omop [Last accessed November 2020].

21. Stang PE, Ryan PB, Racoosin JA, Overhage JM, Hartzema AG, Reich C, et al. Advancing the science for active surveillance: rationale and design for the observational medical outcomes partnership. Ann Intern Med. 2010;153(9):600-6.

22. Observational Health Data Sciences and Informatics, The Book of OHDSI, 2020. Available at: https://ohdsi.github.io/TheBookOfOhdsi/ [Last accessed November 2020]. In.

23. Garza M, Del Fiol G, Tenenbaum J, Walden A, Zozus MN. Evaluating common data models for use with a longitudinal community registry. J Biomed Inform. 2016:64:333-41.

24. Liyanage H, Liaw ST, Jonnagaddala J, Hinton W, de Lusignan S. Common data models (CDMs) to enhance international big data analytics: a diabetes use case to compare three CDMs. Stud Health Technol Inform. 2018;255:60-4.

25. SDTM [https://www.cdisc.org/standards/foundational/sdtm].

26. Matcho A, Ryan P, Fife D, Reich C. Fidelity assessment of a clinical practice research datalink conversion to the OMOP common data model. Drug Saf. 2014;37(11):945-59.

27. Galiè N, Humbert M, Vachiery J-L, Gibbs S, Lang I, Torbicki A, et al. 2015 ESC/ERS Guidelines for the diagnosis and treatment of pulmonary hypertension: The Joint Task Force for the Diagnosis and Treatment of Pulmonary Hypertension of the European Society of Cardiology (ESC) and the European Respiratory Society (ERS): Endorsed by: Association for European Paediatric and Congenital Cardiology (AEPC), International Society for Heart and Lung Transplantation (ISHLT). Eur Heart J. 2015;37(1):67-119.

28. Jansen-van der Weide MC, CMW G, KCB R, Pontes C, Vives R, Sancho $A$, et al. Rare disease registries: potential applications towards impact on development of new drug treatments. Orphanet J Rare Dis. 2018;13(1):154. 
29. Lacaze P, Millis N, Fookes M, Zurynski Y, Jaffe A, Bellgard M, et al. Rare disease registries: a call to action. Intern Med J. 2017:47(9):1075-9.

30. Voss EA, Makadia R, Matcho A, Ma Q, Knoll C, Schuemie M, et al. Feasibility and utility of applications of the common data model to multiple, disparate observational health databases. J Am Med Inform Assoc. 2015;22(3):553-64.

31. Makadia R, Ryan PB. Transforming the Premier Perspective Hospital Database into the Observational Medical Outcomes Partnership (OMOP) Common Data Model. EGEMS (Washington, DC). 2014:2(1):1110.

32. Fischer P, Stöhr MR, Gall H, Michel-Backofen A, Majeed RW. Data integration into OMOP CDM for heterogeneous clinical data collections via HL7 FHIR bundles and XSLT. Stud Health Technol Inform. 2020;270:138-42.

33. Jensen ET, Dabelea DA, Praveen PA, Amutha A, Hockett CW, Isom SP, et al. Comparison of the incidence of diabetes in United States and Indian youth: an international harmonization of youth diabetes registries. Pediatr Diabetes. 2020

34. Belenkaya R, Gurley MJ, Golozar A, Dymshyts D, Miller RT, Williams AE, et al. Extending the OMOP common data model and standardized vocabularies to support observational Cancer research. JCO Clin Cancer Informatics. 2021:5:12-20.

35. Yoon D, Ahn EK, Park MY, Cho SY, Ryan P, Schuemie MJ, et al. Conversion and data quality assessment of electronic health record data at a Korean tertiary teaching hospital to a common data model for distributed network research. Healthc Inform Res. 2016:22(1):54-8.

36. You SC, Lee S, Cho SY, Park H, Jung S, Cho J, et al. Conversion of National Health Insurance Service-National Sample Cohort (NHIS-NSC) database into observational medical outcomes partnership-common data mode (OMOP-CDM). Stud Health Technol Inform. 2017;245:467-70.

37. Ogunyemi OI, Meeker D, Kim HE, Ashish N, Farzaneh S, Boxwala A. Identifying appropriate reference data models for comparative effectiveness research (CER) studies based on data from clinical information systems. Med Care. 2013;51(8 Suppl 3):S45-52.

38. European Health Data Evidence Network. Available at: https://www. ehden.eu/ [Last Accessed June 2021].

39. OHDSI: Chapter 4. The Common Data Model. In: The Book of OHDSI: Observational Health Data Sciences and Informatics. edn.: OHDSI; 2020.

40. Belenkaya R, Gurley M, Dymshyts D, Araujo S, Williams A, Chen R, et al. Standardized observational Cancer research using the OMOP CDM oncology module. Stud Health Technol Inform. 2019;264:1831-2.

41. Warner JL, Dymshyts D, Reich CG, Gurley MJ, Hochheiser H, Moldwin ZH, et al. HemOnc: a new standard vocabulary for chemotherapy regimen representation in the OMOP common data model. J Biomed Inform. 2019;96:103239.

42. Tashkin DP, Amin AN, Kerwin EM. Comparing randomized controlled trials and real-world studies in chronic obstructive pulmonary disease pharmacotherapy. Int J Chron Obstruct Pulmon Dis. 2020;15:1225-43.

43. Negative information in OMOP CDM [https://forums.ohdsi.org/t/negat ive-information-in-omop-cdm/4923].

44. Proposal: Clinical trial data conventions for the OMOP Common Data Model [https://github.com/OHDSI/CommonDataModel/files/4948633/ OMOP.clinical.trial.data.conventions.v1.0.July.2020.pdf].

45. ATLAS - A unified interface for the OHDSI tools [https://www.ohdsi.org/ atlas-a-unified-interface-for-the-ohdsi-tools/].

46. PHederation (Pulmonary Hypertension federated network of real-world assets) [https://portal.phederation.org/].

\section{Publisher's Note}

Springer Nature remains neutral with regard to jurisdictional claims in published maps and institutional affiliations.
Ready to submit your research? Choose BMC and benefit from:

- fast, convenient online submission

- thorough peer review by experienced researchers in your field

- rapid publication on acceptance

- support for research data, including large and complex data types

- gold Open Access which fosters wider collaboration and increased citations

- maximum visibility for your research: over $100 \mathrm{M}$ website views per year

At BMC, research is always in progress.

Learn more biomedcentral.com/submissions 